\title{
Ultrasound morphology of carotid lesions in retinal ischaemia
}

\author{
C M O'Farrell, D E FitzGerald
}

\begin{abstract}
The extacranial carotid arteries of 165 patients with retinal ischaemic symptoms were examined with duplex ultrasound. Both the degree of stenosis and the morphological appearance of the lesions were examined. Ipsilateral carotid artery disease was found in $88 \%$ of patients. Degrees of stenosis of between $50-99 \%$ were found in $33 \%$ of patients, the majority of these lesions being complex heterogeneous in nature. Stenosis of $<50 \%$ was found in $40 \%$ of arteries. The majority of lesions causing $<20 \%$ stenosis were homogeneous in nature. However, in stenosis of $20-49 \%, 63 \%$ of lesions were found to be complex heterogeneous. A similar distribution of lesions was found in the contralateral carotid arteries. It is important to examine both ipsilateral and contralateral arteries, and to evaluate the morphological characteristics of all lesions causing $>\mathbf{2 0 \%}$ stenosis.
\end{abstract}

(Brf Ophthalmol 1992; 76: 656-659)

A relationship between retinal ischaemia and carotid artery disease was first established in 1856 , following autopsy findings of carotid artery thrombosis ipsilateral to retinal ischaemia. ${ }^{1}$ This relationship has been confirmed subsequently. ${ }^{23}$

Diagnostic studies of carotid arterial disease traditionally concentrate on identifying the degree of stenosis caused by atherosclerotic lesions, considering only those which significantly reduce blood flow or perfusion pressure as being potentially clinically important. ${ }^{45}$

It has been shown in patients with amaurosis fugax that there is an increased risk of stroke, blindness and early death in those found to have carotid artery lesions causing even less than $50 \%$ stenosis compared with those patients with no evidence of carotid disease on ultrasound examination, indicating that retinal and cerebral ischaemic symptoms can be caused by carotid lesions previously considered too small to be of clinical significance. ${ }^{6}$ There is evidence that more attention should be given to the ultrasound morphological appearance of atherosclerotic lesions in order to determine their potential clinical significance. ${ }^{78}$ This factor may be as important in producing symptoms as is the amount of stenosis caused by the disease.

The purpose of this report is to give the results of analysis of carotid arterial lesions examined by duplex ultrasound in 165 patients presenting with symptoms of retinal ischaemia. Both the ipsilateral and contralateral carotid arteries have been examined. The atherosclerotic morphological characteristics and degree of stenosis found have been classified in order to try and identify clinically significant disease.

\section{Material and methods}

Between 1985 and 1989, 165 patients (101 male, 64 female, mean age 58 years) were referred to the Diagnostic Blood Flow Laboratory of the Vascular Medicine Unit from an ophthalmic centre for duplex ultrasound examination of the extracranial carotid system. Prior to referral definite retinal ischaemic disease was established on the basis of their ophthalmic assessment, which included funduscopic examination, retinal photography, or fluorescein angiography.

Amaurosis fugax was diagnosed in 79 patients, and 38 patients with permanent unilateral vision loss were found to have central retinal artery occlusion. Branch retinal artery occlusion was found in 48 patients, 17 of whom had partial field defects. Cholesterol emboli were identified in 31 of these patients.

The associated clinical condititions of these patients were: stroke, with a permanent neurological deficit, seven; cerebral transitory ischaemic attack five; peripheral vascular arterial disease 17; ischaemic heart disease 36 . In addition, 62 patients were being treated with anti-hypertensive medication and six patients were on medication for control of diabetes mellitus.

An ATL MK5 duplex ultrasound system with a $7 \cdot 5 \mathrm{MHz}$ imaging facility combined with a 5 $\mathrm{MHz}$ Doppler system was used to examine the extracranial carotid arteries. The examinations were recorded on VHS video tape and hard copies made on a Mitsubishi Printer.

The presence or absence of atherosclerotic lesions was established, and where disease was found the degree of stenosis caused was noted together with a description of the morphological appearance of the lesion. This appearance was described as being either homogeneous or heterogeneous.

Homogeneous lesions had an even echo pattern

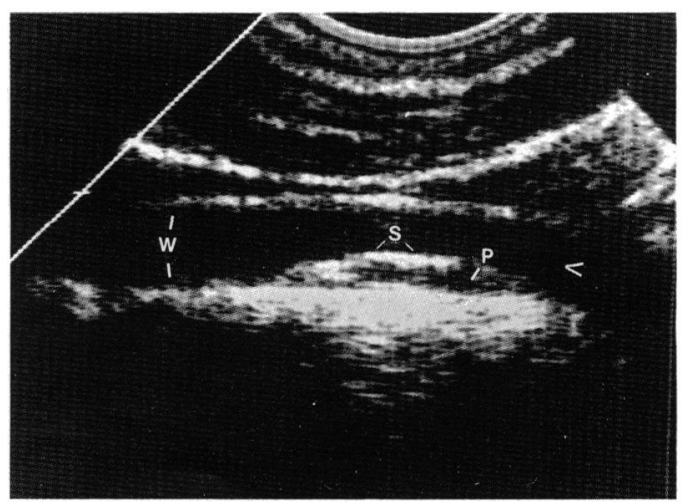

Figure 1 Complex heterogeneous lesion containing a low echo pool $(p)$, without surface irregularity $(s)$. $(w)$ indicates vessel wall and arrowhead direction of blood flow. 


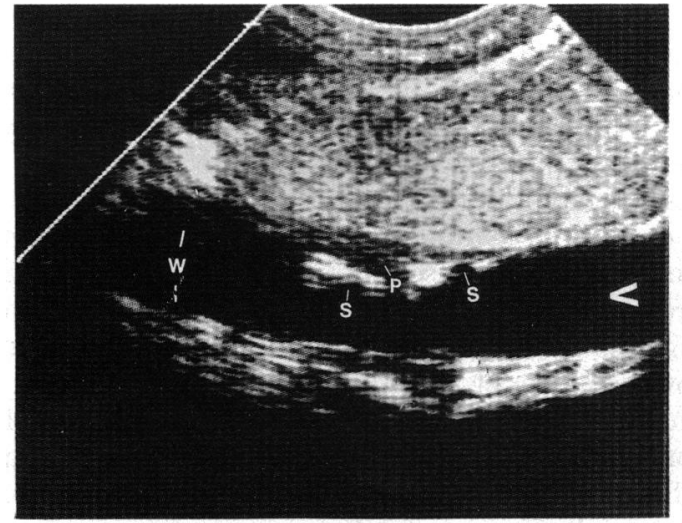

Figure 2 Complex heterogeneous lesion containing low echo pools $(p)$ and having an irregular surface $(s) .(w)$ indicates vessel wall and arrowhead direction of blood flow.

throughout the lesion, and heterogeneous lesions had a mixture of low level and high level echo patterns within the lesion (Figs 1, 2, 3). Heterogeneous structures were further subdivided into simple and complex groups. Simple heterogeneous structures had a smooth surface and a more or less equal distribution of low level and high level echoes. Complex structures displayed low echo pools within the lesions and may also have had an irregular or pitted surface at the interface to flowing blood. The carotid arterial atherosclerosis was classified in terms of degree of stenosis caused and by the morphological characterisation of the lesions.

STATISTICAL ANALYSIS

The $\chi^{2}$ method of statistical analysis was used throughout.

\section{Results}

Atheromatous lesions were found to be present in the ipsilateral ateries of $88 \%$ of the patients presenting with retinal ischaemia (Table 1). Degrees of stenosis of between $50-99 \%$ were found in $33 \%$ of patients, and a further $5 \%$ of patients presented with total occlusion of the internal carotid artery. Lesions causing less than $50 \%$ stenosis were found in $40 \%$ of ipsilateral carotid arteries.

The distribution of homogeneous and heterogeneous lesions in ipsilateral and contralateral arteries is shown in Table 2.

There was a significantly greater incidence of heterogeneous than of homogeneous lesions in both ipsilateral and contralateral arteries $(\mathrm{p}<0.001)$. The incidence of complex heterogeneous lesions was significantly greater than simple heterogeneous lesions $(\mathrm{p}<0.001)$; however, there was no difference in incidence of lesions between the ipsilateral and contralateral arteries.

Table 3 shows the relationship between degrees of stenosis and morphological structure. A homogeneous structure was found predominantly in lesions of less than $20 \%$ stenosis.

In stenosis of more than $50 \%$ all lesions were found to be heterogeneous and of these $82 \%$ were complex. In stenoses of $20-49 \%$ the majority of lesions were heterogeneous and, of these, $63 \%$ were complex in nature.

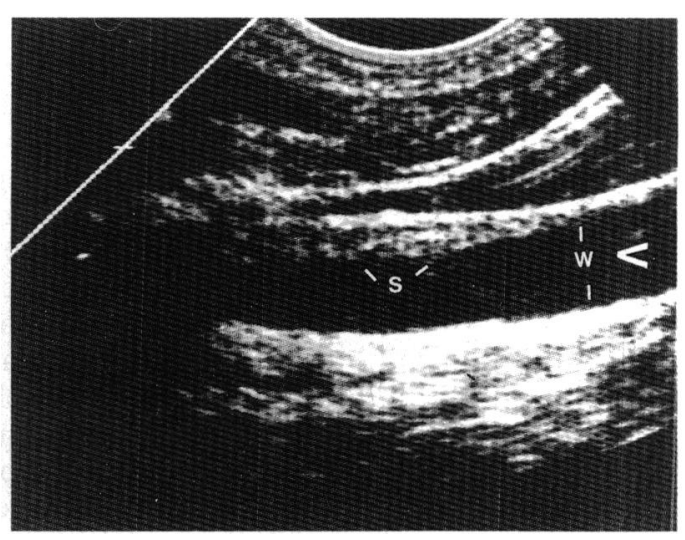

Figure 3 Homogeneous lesion with smooth surface (s). (w) indicates vessel wall and arrowhead direction of blood flow.

\section{Discussion}

The association between severe carotid artery disease and retinal ischaemic events has been well documented. ${ }^{23}$

In this study, the relationship between lesion morphological ultrasound characteristics and patient symptoms was investigated. Lesions were classified as being homogeneous or heterogeneous according to well established criteria..$^{910}$ Heterogeneous lesions were further subdivided into simple or complex appearances, the latter either containing low echo pools or having an irregular surface pattern. The degree of stenosis caused by the lesion was also established. ${ }^{.1}$ Image quality and reproducibility are dependent on machine characteristics. ${ }^{12} 13$ In this study the same duplex ultrasound system was used for all scans.

A significant number of patients presenting

Table 1 The degree of stenosis in the ipsilateral and contralateral carotid arteries

\begin{tabular}{llllll}
\hline & Clear & $1-19 \%$ & $20-49 \%$ & $50-99 \%$ & $\begin{array}{l}\text { Total } \\
\text { occlusion }\end{array}$ \\
\hline Ipsilateral & 38 & 24 & 43 & 57 & 9 \\
Contralateral & 36 & 35 & 36 & 51 & 1 \\
\hline
\end{tabular}

There is no significant difference in the distribution of lesions between the ipsilateral and contralateral carotid arteries.

Table 2 The morphology of lesions in the ipsilateral and contralateral carotid arteries

\begin{tabular}{llll}
\hline & Homogeneous & $\begin{array}{l}\text { Simple } \\
\text { heterogeneous }\end{array}$ & $\begin{array}{l}\text { Complex } \\
\text { heterogeneous }\end{array}$ \\
\hline Ipsilateral & 23 & 23 & 78 \\
Contralateral & 23 & 34 & 65
\end{tabular}

There is a significant difference in distribution between homogeneous and heterogeneous lesions $(p<0.001)$, and between simple heterogeneous and complex heterogeneous lesions $(p<0.001)$. However, no difference in lesion distribution was found between the ipsilateral and contralateral arteries.

Table 3 The relationship between lesion morphology and degree of stenosis in the ipsilateral carotid arteries

\begin{tabular}{llll}
\hline $\begin{array}{l}\text { Degree of } \\
\text { stenosis }(\%)\end{array}$ & Homogeneous & $\begin{array}{l}\text { Simple } \\
\text { heterogeneous }\end{array}$ & $\begin{array}{l}\text { Complex } \\
\text { heterogeneous }\end{array}$ \\
\hline $1-19$ & 18 & 2 & 4 \\
$20-49$ & 5 & 11 & 27 \\
$60-99$ & 0 & 10 & 47 \\
\hline
\end{tabular}

The majority of lesions causing $<20 \%$ are homogeneous and those causing $>60 \%$ stenosis complex heterogeneous. In the $20-49 \%$ stenosis group, the majority of lesions are heterogeneous, and of those $63 \%$ are complex in nature. 
with retinal ischaemia, whose carotid artery disease might not have been considered clinically significant in terms of degree of stenosis, were found to have complex heterogeneous lesions. This suggests that complex heterogeneous lesions may be potential sources of emboli even when occupying less than $50 \%$ of the arterial lumen.

Several studies have compared the ultrasound characterisation of atherosclerotic plaques with their histological examination. Homogeneous plaques have been shown to consist of predominantly fibrous material, and have a poor association with symptom production. Heterogeneous plaques have been found to contain either intraplaque haemorrhage or lipid products, and while ultrasound cannot distinguish between these materials, both have been strongly associated with ischaemic symptoms. ${ }^{7810121416}$

Considerable variation has been reported in the accuracy of ultrasonic diagnosis of ulceration. ${ }^{12}{ }^{17-22}$ However, as it is the acoustic surface of the lesion that is demonstrated by ultrasound, it is more accurate to describe the characteristics of the plaque surface rather than use the term ulceration, which has a specific pathological definition.

Both ulcerative plaques and lesions with surface irregularities have a reported association with ischaemic symptoms. ${ }^{22} 2324$

In a recent study of 108 patients with suspected carotid atherosclerosis, it was found that it is the heterogeneous nature of the lesion, rather than its size, that is associated with clinical symptoms. Duplex ultrasound of the carotid arteries did not reveal a correlation between degree of stenosis and hemispheric symptoms. However, significant correlation was found between heterogeneous plaques and hemispheric symptoms. The authors noted that the majority of plaques causing $>50 \%$ stenosis were heterogeneous in nature. ${ }^{9}$

A strong association between complex heterogeneous lesions and retinal ischaemic symptoms has been found in this study. There is a known association between severe stenosis and the production of symptoms, and lesions causing severe stenosis were usually found to be complex and heterogeneous in nature.

In this study of patients presenting with symptomatic retinal ischaemia, $40 \%$ of patients were found to have relatively small degrees of stenosis, but quite a high proportion of these smaller lesions were also complex and heterogeneous in structure. This emphasises the clinical importance of the ultrasound morphology of atherosclerotic lesions.

Clinical management of carotid artery disease is still controversial. The recent MRC European Carotid Surgery Trial report indicates benefit from surgery in those patients preseriting with $70-99 \%$ stenosis, and does not recommend surgery for less than $30 \%$ stenosis. The findings for those with stenoses greater than $30 \%$ and less than $70 \%$ are still awaited..$^{25}$ It should be noted that this trial did not take account of lesion morphology.

The role of anti-platelet therapy is widely established, and has been shown to reduce vascular mortality by $15 \%$ and non-fatal vascular events by $30 \%$ in patients with a history of cerebrovascular or cardiovascular disease. ${ }^{26}$

The non-invasive nature of ultrasound allows investigation of the contralateral asymptomatic arteries. This study shows an unexpectedly similar distribution of severity of lesions in both the ipsilateral and contralateral carotid arteries. There is a trend towards a higher incidence of more severe lesions on the ipsilateral side, a finding which has been observed by two previous reports. ${ }^{27} 28$

A similar morphological pattern of atherosclerotic lesions was also noted on both arterial sides. It has been suggested that the type of carotid disease which gives rise to retinal ischaemic symptoms and that which results in hemispheric transient ischaemic attacks may be different, the former producing small emboli which also cause multiple silent infarcts confirmed by computed tomographic scan, with an associated increased incidence of cerebral atrophy. ${ }^{29}$ The similarity of distribution of lesions in the ipsilateral and contralateral sides in this study also raises the possibility that silent cerebral events may be occurring without involving the contralateral retina.

For this reason it is recommended that both carotid vessels be examined routinely as there is a potential danger of emboli from the contralateral side affecting either the retina or causing silent cerebral infarction.

All the patients in this study were referred by an ophthalmic centre specifically for carotid ultrasound studies. The results contain valuable information for the ophthalmic management of these patients. As a result the ophthalmologist may be the first professional to receive information about potential risk not only to the eye but also to the heart and brain.

1 Virchow R. Zur Pathologist Anatomie Der Netzhaut und Sehnerven. Virchows Arch [Cell Pathol] 1856; 10: 170-93.

2 Kollaritis CR, Lubow M, Hissong S. Retinal strokes 1. Incidence of carotid atheroma. FAMA 1972; 222: 1273-5.

3 Chawluk JB, Kushner MJ, Bank WJ, Silver FL, Jamieson DG, Bosley TM, et al. Atheromatous carotid artery disease in patients with retinal ischaemic syndromes. Neurology in patients with

4 Riles TS, Liberman A, Kopelman I, Imperato AM. Symptoms, stenosis and bruit: inter-relationships in carotid artery disease. Arch Surg 1981; 116: 218-20.

5 Boujousslavski J, Despland P, Regli F. Asymptomatic tight stenosis of the internal carotid artery: long term prognosis Neurology 1986; 36: 861-3.

6 Torem S, Rossman ME, Schneider PA, Otis SM, Dilley RB Bernstein EF. The natural history of amaurosis fugax with minor degrees of internal carotid stenosis. Ann Vasc Surg $1990 ; 4: 46-51$.

7 Gray-Weale AC, Graham JC, Burnett JR, Byrne K, Lusby RJ Carotid artery atheroma: comparison of pre operative $\mathrm{B}$ mode ultrasound appearance with carotid endarterectomy mode ultrasound appearance with carotid endarterectom

8 Bluth EI, Kay D, Merritt CKB, Sullivan M, Farr G, Mills NL, et al. Sonographic characterisation of carotid plaque: detection of haemorrhage. AfR 1986; 146: 1061-5.

9 Leahy AL, McCollum PT, Feeley TM, Sugrue M, Grouden $\mathrm{MC}$, O'Connell DJ, et al. Duplex ultrasonography and selection of patients for carotid endarterectomy: plaque morphology or luminal narrowing? $\mathcal{F}$ Vasc Surg 1988; 8 558-62.

10 Reilly LM, Lusby RJ, Hughes L, Ferrell LD, Stoney RJ, Ehrenfeld WK. Carotid plaque histology using real time ultrasonography. Am F Surg 1983; 146: 188-93.

11 Taylor D, Strandness DE Jr. Carotid artery duplex scanning. f Clin Ultrasound 1987; 15: 635-44.

12 Widder B, Paulat K, Hackspacher J, Hamann H, Hutschenr $\mathrm{S}$, Kruentzer C, et al. Morphological characterisation of carotid artery stenosis by ultrasound duplex scanning. carotid artery stenosis by ultrasound
Ultrasound Med Biol 1990; 16: 349-54.

13 Labs KH, Jäger K, FitzGerald DE, Woodcock JP, NeuerburgHeusler D, eds. Diagnostic vascular ultrasound. London: Heusler D, eds. Diagnostic vasc
Edward Arnold, 1992: 321-5.

14 Goes E, Janssens W, Maillet B, Freson M, Steyaert L, Osteaux $M$. Tissue characterisation of atheromatous plaques: 
correlation between ultrasound image and histological findings. 7 Clin Ultrasound 1990; 18: 611-7.

15 Langsfield M, Gray-Weale AC, Lusby RJ. The role of plaque morphology and diameter reduction in the development of new symptoms in asymptomatic carotid arteries. $\mathcal{F}$ Vasc Surg 1989; 9: 548-56.

16 Widder B. Impact of ultrasound methods on decision making for carotid and EC/IC by-pass surgery. In: Aichner F, Gerstenbrand F, Greevic N, eds. The present state in clinical neuro-imaging. New York: Gustav Fischer, 1989: 275-80.

17 Rubin J, Bondi J, Rhodes R. Duplex scanning versus conventional arteriography for the evaluation of carotid artery plaque morphology. Surgery 1987; 102: 749-55.

18 O'Donnell T, Erodes L, Mackey W, McCullough J, Shephard $\mathrm{A}$, Heggerick $\mathrm{P}$, et al. Correlation of B-mode ultrasound imaging and arteriography with pathological findings at carotid endarterectomy. Arch Surg 1985; 120: 443-9.

19 Bluth E, McVay L, Merritt C, Sullivan M. The identification of ulcerative plaque with high resolution duplex carotid of ulcerative plaque with high resolution
scanning. $\mathcal{F}$ Ultrasound Med $1988 ; 7: 73-6$.

20 Davenport K, Sterpetti A Hunter W, Schultz R, Feldhaus R di Marzo L, et al. Real-time B-mode carotid imaging and di Marzo L, et al. Real-time B-mode carotid imagint

21 O'Leary D, Holen J, Ricotta J, Roe S, Schenk E. Carotid bifurcation disease: prediction of ulceration with B-mode US. Radiology 1987; 162: 523-5.

22 Sterpetti A, Hunter W, Schultz R. Importance of ulceration of carotid plaque in determining symptoms of cerebral ischaemia. F Cardiovasc Surg 1991; 32: 154-8.
23 Imperato A, Riles T, Mintzer R, Baumann G. The importance of haemorrhage in the relationship between gross morphological characteristics and cerebral symptoms in 376 carotid artery plaques. Ann Surg 1983; 197: 195-203.

24 Dixon S, Osher S, Raviola C, Gomes A, Machleder H, Baker JD, et al. Natural history of non-stenotic, asymptomatic JD, et al. Natural history of non-stenotic, asymptomatic 1493-8.

25 European Carotid Surgery Trialist Collaborative Group. MRC European Carotid Surgery Trial: interim results for symptomatic patients with severe $(70-99 \%)$ or with mild $(0-29 \%)$ carotid stenosis. Lancet 1991; 337: 1235-43.

26 Anti-platelet Trialists Collaboration. Secondary prevention of vascular disease by prolonged anti-platelet treatment. $B M \mathcal{F}$ 1988; 296: 320-31.

27 Hankey GJ, Slatter JM, Warlow GP. Prognosis and prognostic factors of retinal infarction: a prospective cohort study. BMF 1991; 302: 499-504.

28 Stavenow L, Bjerre P, Lindgarde F. Patients with reversible ischaemic neurological deficit have less severe carotid artery lesions than those with transient ischaemic carotid artery lesions than those with transient ischaemic 7: $32-6$.

29 Grigg MJ, Papadakis K, Nicolaides AN, Al-Kutonbi A, Williams MA, Deacon DFS, et al. The significance of cerebral infarction and atrophy in patients with amaurosis fugax and transient ischaemic attacks in relation to internal carotid artery stenosis: a preliminary report. $\mathcal{F}$ Vasc Surg 1988; 7 : 215-21. 